\title{
51
}

\section{Verhaltensbiologie (Multiple Choice)}

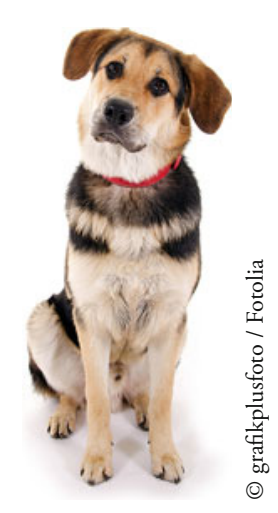

Woher weiß die Katze eigentlich, wie sie zu jagen hat? Was bedeutet es, instinktiv zu handeln? Wähle die jeweils richtige Antwort aus und bilde das Lösungswort aus den erhaltenen Buchstaben.

1. Was ist keine Phase einer Instinkthandlung?

(E) Appetenz (C) Reflex (A) Endhandlung

2. Voraussetzung zum Auslösen eines bestimmten Instinktverhaltens

(L) Initialreiz (M) Auslösereiz (U) Schlüsselreiz

3. Lernprozess, bei dem ein Reiz mit einer Reaktion verknüpft wird

(R) Konditionierung (L) Reflexierung (P) Evolution

4. Form des Lernens, bei der Erfahrungen in erbliche Verhaltensmuster eingebaut werden
(A) Prägung
(E) Trauma
(I) Doktorarbeit

5. Paarungssystem, bei dem ein Männchen mehrere Weibchen hat (I) Monogamie (E) Polyandrie (R) Polygynie

6. Das Laubenvogel-Männchen schmückt seinen Balzplatz mit...

(E) blauen Gegenständen ( $\mathbf{T}$ ) seinen Federn (S) erbeuteten Schmetterlingen

Lösung:

(C) Springer-Verlag Berlin Heidelberg 2016

C. Reinbold, Fetthenne, Moderlieschen, Warzenbeißer,

DOI 10.1007/978-3-662-52817-4_51 